\title{
ANALYSIS OF LOCALLY STABILIZED MIXED FINITE ELEMENT METHODS FOR THE STOKES PROBLEM
}

\author{
NASSERDINE KECHKAR AND DAVID SILVESTER
}

\begin{abstract}
In this paper, a locally stabilized finite element formulation of the Stokes problem is analyzed. A macroelement condition which is sufficient for the stability of (locally stabilized) mixed methods based on a piecewise constant pressure approximation is introduced. By satisfying this condition, the stability of the $Q_{1}-P_{0}$ quadrilateral, and the $P_{1}-P_{0}$ triangular element, can be established.
\end{abstract}

\section{INTRODUCTION}

The development of stable finite element methods for the Stokes equations is a fundamental component in the search for efficient numerical methods for solving the Navier-Stokes equations governing the flow of an incompressible fluid. For a primitive variable formulation, the importance of ensuring the compatibility of the component approximations of velocity and pressure by satisfying the so-called "Babuška-Brezzi stability condition" is widely understood. In particular, it is well known that conforming low-order elements like the $P_{1}-P_{0}$ (linear velocity, constant pressure) triangle are not stable. This impinges on efficiency, since the simple logic and regular data structure associated with low-order finite element methods make them particularly attractive on modern vector and parallel processing architectures.

The stability of the mixed approximations has become crucially important with the advent of "fast" iterative solution algorithms, for example, based on multigrid or preconditioned conjugate gradient iterations. Numerical experiments show that in the solution of the Stokes or Navier-Stokes equations, ensuring stability is essential if a reasonable rate of convergence of such iterations is to be achieved. For details, see the recent work of Verfürth [16], and Bramble and Pasciak [4].

Recently, regularizations of the discrete Stokes formulation have been developed as a means of overcoming the problem of incompatible mixed approximations. The idea of such a regularization was first proposed by Brezzi and Pitkäranta [5] in the context of the $P_{1}-P_{1}$ triangular element. Subsequently, Hughes and Franca [9] derived a discrete Stokes formulation which ensured the stability of arbitrary mixed approximations. For a discontinuous pressure approximation, this stability is achieved by introducing a pressure jump operator

Received August 9, 1989; revised June 21, 1990 and October 12, 1990.

1991 Mathematics Subject Classification. Primary 65N15, 65N30.

Key words and phrases. Stabilized finite element, Stokes equation. 
into the discrete formulation. For low-order approximations, the only price to pay for having universal stability is that the jump operator must control pressure jumps across all internal interelement edges. This makes the Hughes and Franca formulation awkward to implement, since a nonstandard element assembly algorithm is required. We note that this limitation also applies to the absolutely stable formulation recently proposed by Douglas and Wang [6].

Numerically, there is evidence (see, for example, [14]) to suggest that a more robust way of stabilizing a mixed method based on discontinuous pressure is to modify the "global" jump operator of Hughes and Franca, so as to restrict the jumps in pressure in a "local" sense. In [14] we refer to the original and the modified formulations as global jump and local jump stabilizations, respectively. The precise definitions are given in the next section. A key feature of a local jump stabilization is that a conventional macroelement implementation is possible, so that the modified methods can be directly implemented into element-by-element iterative solution techniques.

In this paper we aim to derive a general method of analysis for such locally stabilized mixed approximations. At the heart of the analysis is a local "macroelement condition," which is sufficient for the overall stability of the method. The use of such a macroelement condition as a means of verifying the Babuška-Brezzi stability inequality is standard practice (see, for example, Girault and Raviart [7, p. 129]. The basic idea was first introduced by Boland and Nicolaides [3], and independently by Stenberg [15]. For ease of notation, and to keep the paper brief, we will confine our attention to the $Q_{1}-P_{0}$ (bilinear velocity, constant pressure) quadrilateral, and the $P_{1}-P_{0}$ triangular element. The generalization of our analysis to cover higher-order locally stabilized methods, and three-dimensional tetrahedral and hexahedral elements, is straightforward.

An outline of the paper is as follows. In $\S 2$ the global and the locally stabilized Stokes formulations are defined. In $\S 3$ the macroelement condition, which is the key to stability, is introduced, and error estimates for the two locally stabilized methods are obtained. By comparing these estimates with those of the globally stabilized method, the improved robustness of the local stabilization approach is justified theoretically.

\section{Stabilized Stokes Formulations}

With the usual notation, i.e., defining the velocity and pressure spaces by $\mathbf{V} \equiv$ $\left[H_{0}^{1}(\Omega)\right]^{2}$ and $P \equiv L_{0}^{2}(\Omega)$, respectively, the classical variational formulation of the Stokes equation (cf. [7, p. 82]) is: find $\mathbf{u} \in \mathbf{V}$ and $p \in P$ such that

$$
\begin{aligned}
(\operatorname{grad} \mathbf{u}, \operatorname{grad} \mathbf{v})-(p, \operatorname{div} \mathbf{v}) & =(\mathbf{f}, \mathbf{v}) & & \forall \mathbf{v} \in \mathbf{V}, \\
(q, \operatorname{div} \mathbf{u}) & =0 & & \forall q \in P,
\end{aligned}
$$

where $(\cdot, \cdot)$ denotes the usual $L^{2}$ inner product.

Introducing the generalized bilinear form

$$
\mathscr{B}((\mathbf{u}, p) ;(\mathbf{v}, q))=(\operatorname{grad} \mathbf{u}, \operatorname{grad} \mathbf{v})-(p, \operatorname{div} \mathbf{v})-(q, \operatorname{div} \mathbf{u})
$$

and the linear functional

$$
\mathscr{L}(\mathbf{v}, q)=(\mathbf{f}, \mathbf{v}),
$$

we can recast the Stokes formulation (2.1) as: find $(\mathbf{u}, p) \in \mathbf{V} \times P$ such that

$$
\mathscr{B}((\mathbf{u}, p) ;(\mathbf{v}, q))=\mathscr{L}(\mathbf{v}, q) \quad \forall(\mathbf{v}, q) \in \mathbf{V} \times P .
$$


Finite element subspaces of $\mathbf{V}$ and $P$ are characterized by $\tau_{h}$, a partitioning of $\bar{\Omega}$ into triangles or quadrilaterals, assumed to be regular in the usual sense, i.e., for some $\sigma$ and $\omega$ with $\sigma>1$ and $0<\omega<1$,

$$
\begin{gathered}
h_{K} \leq \sigma \rho_{K} \quad \forall K \in \tau_{h}, \\
\left|\cos \theta_{i K}\right| \leq \omega, \quad i=1,2,3,4, \quad \forall K \in \tau_{h},
\end{gathered}
$$

where $h_{K}$ is the diameter of element $K, \rho_{K}$ is the diameter of the inscribed circle of element $K$, and $\theta_{i K}$ are the angles of $K$ in the case of a quadrilateral partitioning. The mesh parameter $h$ is given by $h=\max \left(h_{K}\right)$, and the set of all interelement boundaries will be denoted by $\Gamma_{h}$.

The finite element subspaces of interest in this paper are defined by setting

$$
R_{1}(K)= \begin{cases}P_{1}(K) & \text { if } K \text { is triangular, } \\ Q_{1}(K) & \text { if } K \text { is quadrilateral, }\end{cases}
$$

giving the continuous piecewise (bi)linear velocity subspace

$$
\mathbf{V}_{h}=\left\{\mathbf{v}=\left(v_{1}, v_{2}\right) \in\left[H_{0}^{1}(\Omega)\right]^{2} ; v_{i_{\mid K}} \in R_{1}(K), i=1,2, \forall K \in \tau_{h}\right\}
$$

and the piecewise constant pressure subspace

$$
P_{h}=\left\{q \in L_{0}^{2}(\Omega) ; q_{\mid K} \in P_{0}(K), \forall K \in \tau_{h}\right\} .
$$

We note that neither of these methods are stable in the standard BabuškaBrezzi sense; the $P_{1}-P_{0}$ triangle "locks" on regular grids (since there are more discrete incompressibility constraints than velocity degrees of freedom), the $Q_{1}-P_{0}$ quadrilateral is the most infamous example of an unstable mixed method, as elucidated by Sani et al. [13].

With the choices of $\mathbf{V}_{h}$ and $P_{h}$ above, a globally stabilized discrete formulation of the Stokes problem (cf. [9]) is: find $\mathbf{u}_{h} \in \mathbf{V}_{h}$ and $p_{h} \in P_{h}$ such that

$$
\begin{array}{rlrl}
\left(\operatorname{grad} \mathbf{u}_{h}, \operatorname{grad} \mathbf{v}\right)-\left(p_{h}, \operatorname{div} \mathbf{v}\right) & =(\mathbf{f}, \mathbf{v}) & & \forall \mathbf{v} \in \mathbf{V}_{h}, \\
\left(q, \operatorname{div} \mathbf{u}_{h}\right)+\beta \sum_{e \in \Gamma_{h}} h_{e} \int_{e} \llbracket p_{h} \rrbracket_{e} \llbracket q \rrbracket_{e} d s=0 & & \forall q \in P_{h},
\end{array}
$$

where $\llbracket \cdot \mathbb{l}_{e}$ is the jump operator across $e \in \Gamma_{h}$ and $\beta>0$ is the "global" stabilization parameter.

Given any subdivision $\tau_{h}$, a macroelement partitioning $\mathscr{M}_{h}$ may be defined such that each macroelement $M$ is a connected set of adjoining elements from $\tau_{h}$. Every element $K$ must lie in exactly one macroelement, which implies that macroelements do not overlap. For each $M$, the set of interelement edges which are strictly in the interior of $M$ will be denoted by $\Gamma_{M}$. Furthermore, the length of edge $e \in \Gamma_{M}$ is denoted by $h_{e}$.

With these additional definitions a locally stabilized discrete formulation of the Stokes problem (2.1) can be stated: find $\mathbf{u}_{h} \in \mathbf{V}_{h}$ and $p_{h} \in P_{h}$ such that

$$
\begin{gathered}
\left(\operatorname{grad} \mathbf{u}_{h}, \operatorname{grad} \mathbf{v}\right)-\left(p_{h}, \operatorname{div} \mathbf{v}\right)=(\mathbf{f}, \mathbf{v}) \quad \forall \mathbf{v} \in \mathbf{V}_{h}, \\
\left(q, \operatorname{div} \mathbf{u}_{h}\right)+\beta \sum_{M \in \mathscr{h}_{h}} \sum_{e \in \Gamma_{M}} h_{e} \int_{e} \llbracket p_{h} \rrbracket_{e} \llbracket q \rrbracket_{e} d s=0 \quad \forall q \in P_{h},
\end{gathered}
$$

where $\llbracket \cdot \mathbb{l}_{e}$ is the jump operator across $e \in \Gamma_{M}$ and $\beta>0$ is the "local" stabilization parameter. 


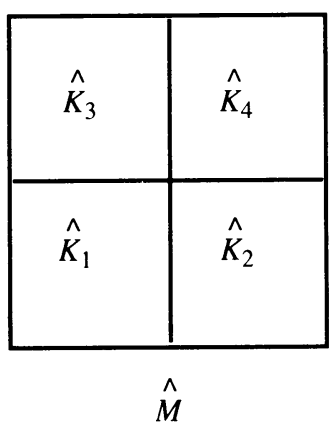

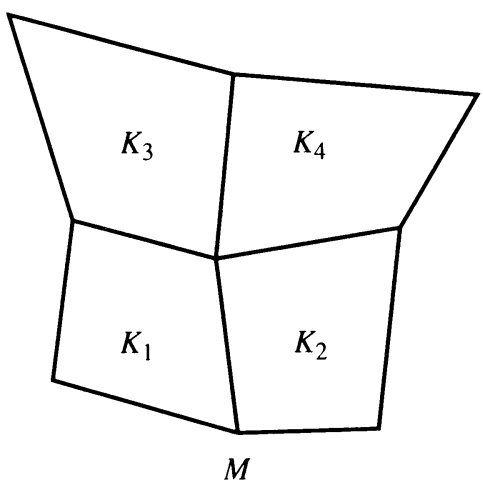

FIGURE 1

Reference and arbitrary $\left(Q_{1}-P_{0}\right)$ macroelements

A general framework for analyzing the locally stabilized formulation (2.11) can be developed using the notion of equivalence classes of macroelements. As in Stenberg [15], each equivalence class, denoted by $\mathscr{E}_{\widehat{M}}$, contains macroelements which are topologically equivalent to a reference macroelement $\widehat{M}$. To illustrate the idea, two practical examples of locally stabilized mixed approximations are given below.

Example 2.1. The first example is the standard $Q_{1}-P_{0}$ approximation pair. A locally stabilized formulation (2.11) can be constructed in this case, if $\tau_{h}$ is such that the elements can be grouped into $2 \times 2$ macroelements, with the reference macroelement $\widehat{M}$ and an arbitrary $M \in \mathscr{E}_{\widehat{M}}$ as illustrated in Figure 1 (there is only one equivalence class in this case).

An obvious way of constructing such a partitioning in practice is to form the grid $\tau_{h}$ by uniformly refining a coarse grid $\mathscr{M}_{h}$, for example, by joining the mid-edge points.

Example 2.2. The triangular $P_{1}-P_{0}$ approximation pair can similarly be stabilized if the partitioning $\tau_{h}$ is constructed such that the elements can be grouped into disjoint macroelements, all consisting of four elements, as illustrated in Figure 2.

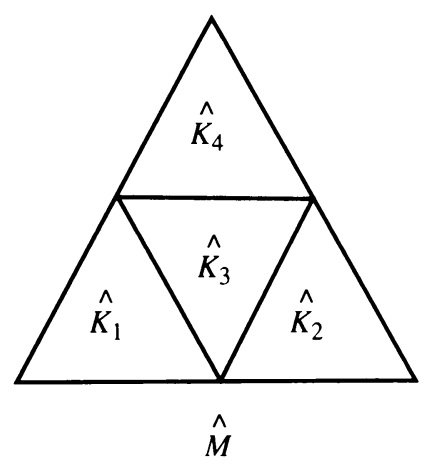

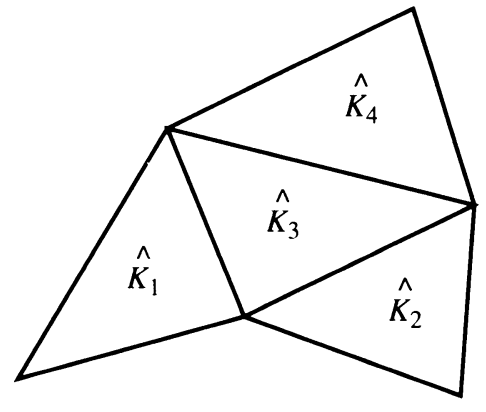

$M$

FigURE 2

Reference and arbitrary $\left(P_{1}-P_{0}\right)$ macroelements 
The stability of these mixed methods for the macroelement partitionings defined above are formally established in the next section.

\section{Stability OF THE LOCAlly STABilized StOKes FoRMUlation}

For a macroelement $M$ the restricted pressure space is given by

$$
P_{0, M}=\left\{q \in L_{0}^{2}(M) ; q_{\mid K} \text { is constant } \forall K \subset M\right\} .
$$

Defining a symmetric bilinear form $\mathscr{C}_{h}(\cdot, \cdot)$, which is defined on $P_{h} \times P_{h}$, by

$$
\mathscr{C}_{h}(r, q)=\sum_{M \in \mathscr{M}_{h}} \sum_{e \in \Gamma_{M}} h_{e} \int_{e} \llbracket r \rrbracket_{e} \llbracket q \rrbracket_{e} d s,
$$

we first prove a local coercivity result for the form $\mathscr{C}_{M}(\cdot, \cdot)$ which is the restriction of $\mathscr{C}_{h}(\cdot, \cdot)$ to a macroelement $M$, i.e.,

$$
\mathscr{C}_{M}(r, q)=\sum_{e \in \Gamma_{M}} h_{e} \int_{e} \llbracket r \rrbracket_{e} \llbracket q \rrbracket_{e} d s .
$$

Lemma 3.1. Let $\mathscr{E}_{\widehat{M}}$ be a class of macroelements. Then there is a positive constant $\gamma_{\widehat{M}}=\gamma(\widehat{M})$ such that

$$
\mathscr{C}_{M}(q, q) \geq \gamma_{\widehat{M}}\|q\|_{0, M}^{2} \quad \forall q \in P_{0, M}
$$

holds for every $M \in \mathscr{E}_{\widehat{M}}$.

Proof. Consider first a fixed $M \in \mathscr{E}_{\widehat{M}}$. From the definition of $\mathscr{C}_{M}$ we note that $\mathscr{C}_{M}(q, q)=0$ if and only if $q_{\mid M}$ is a constant. Hence, the constant $\gamma_{M}$ defined through

$$
\gamma_{M}=\inf _{\substack{q \in P_{0, M} \\\|q\|_{0, M}=1}} \mathscr{C}_{M}(q, q)
$$

is positive. Next, by virtue of a scaling argument (cf. [15, Lemma 3.1]), the regularity conditions (2.5) and (2.6) ensure that there is a constant $\gamma_{\widehat{M}}$ such that

$$
\gamma_{M} \geq \gamma_{\widehat{M}}>0 \quad \forall M \in \mathscr{E}_{\widehat{M}},
$$

which implies (3.3).

Let us assume now that there is a fixed set of classes $\mathscr{E}_{\widehat{M}_{i}}, i=1, \ldots, n$, $n \geq 1$, such that every macroelement $M \in \mathscr{M}_{h}$ belongs to one of the equivalence classes.

Following [15], we define $\Pi_{h}$ to be the $L^{2}$ projection from $P_{h}$ onto the subspace

$$
Q_{h}=\left\{\mu \in L_{0}^{2}(\Omega) ; \mu_{\mid M} \text { is constant } \forall M \in \mathscr{M}_{h}\right\} .
$$

A direct consequence of the lemma is the following "global" inequality:

$$
\mathscr{C}_{h}(q, q) \geq \alpha_{1}\left\|\left(I-\Pi_{h}\right) q\right\|_{0}^{2} \quad \forall q \in P_{h},
$$

where $\alpha_{1}=\min \left\{\gamma_{\widehat{M}_{i}}, i=1, \ldots, n\right\}$ and is independent of $h$. 
The locally stabilized formulation (2.11) can be written in the general form: given $\beta>0$, find $\mathbf{u}_{h} \in \mathbf{V}_{h}, p_{h} \in P_{h}$ such that

$$
\mathscr{B}_{h}\left(\left(\mathbf{u}_{h}, p_{h}\right) ;(\mathbf{v}, q)\right)=\mathscr{L}(\mathbf{v}, q) \quad \forall(\mathbf{v}, q) \in \mathbf{V}_{h} \times P_{h},
$$

where

$$
\begin{aligned}
\mathscr{B}_{h}\left(\left(\mathbf{u}_{h}, p_{h}\right) ;(\mathbf{v}, q)\right)= & \left(\operatorname{grad} \mathbf{u}_{h}, \operatorname{grad} \mathbf{v}\right)-\left(p_{h}, \operatorname{div} \mathbf{v}\right) \\
& -\left(q, \operatorname{div} \mathbf{u}_{h}\right)-\beta \mathscr{C}_{h}\left(p_{h}, q\right) .
\end{aligned}
$$

The main result of the paper is now stated and proved.

Theorem 3.1. Given a stabilization parameter $\beta \geq \beta_{0}>0$, suppose that every macroelement $M \in \mathscr{M}_{h}$ belongs to one of the equivalence classes $\mathscr{E}_{\widehat{M}_{i}}$, and that the following macroelement connectivity condition is valid: for any two neighboring macroelements $M_{1}$ and $M_{2}$ with $\int_{M_{1} \cap M_{2}} d s \neq 0$ there exists $\mathbf{v} \in \mathbf{V}_{h}$ such that

$$
\text { supp } \mathbf{v} \subset M_{1} \cup M_{2} \text { and } \int_{M_{1} \cap M_{2}} \mathbf{v} \cdot \mathbf{n} d s \neq 0 .
$$

Then, assuming that $\mathbf{u} \in\left[H^{2}(\Omega)\right]^{2}$ and $p \in H^{1}(\Omega)$, there exists a constant $C$, independent of $\beta$, such that we have the error estimate

$$
\left\|\mathbf{u}-\mathbf{u}_{h}\right\|_{1}+\left\|p-p_{h}\right\|_{0} \leq C h\left(|\mathbf{u}|_{2}+|p|_{1}\right) .
$$

Proof. Following Babuška [1], we first establish the existence of a constant $\alpha>$ 0 , independent of $\beta$, such that for all $(\mathbf{v}, q) \in \mathbf{V}_{h} \times P_{h}$

$$
\sup _{(\mathbf{w}, r) \in \mathbf{V}_{h} \times P_{h}} \frac{\mathscr{B}_{h}((\mathbf{v}, q) ;(\mathbf{w}, r))}{\|\mathbf{w}\|_{1}+\|r\|_{0}} \geq \alpha\left(\|\mathbf{v}\|_{1}+\|q\|_{0}\right) .
$$

To this end, let $(\mathbf{v}, q) \in \mathbf{V}_{h} \times P_{h}$ and $\alpha_{1}$ be as above. Because of the condition (3.8) (cf. [15, Lemma 3.3]), there exist a positive constant $\alpha_{2}$, independent of $h$, and a $\mathbf{g} \in \mathbf{V}_{h}$ satisfying

$$
\left(\Pi_{h} q, \operatorname{div} \mathbf{g}\right)=\left\|\Pi_{h} q\right\|_{0}^{2} \text { and }\|\mathbf{g}\|_{1} \leq \alpha_{2}\left\|\Pi_{h} q\right\|_{0} .
$$

If $\mathbf{w} \in \mathbf{V}_{h}$ and $r \in P_{h}$ are now chosen such that $\mathbf{w}=\mathbf{v}-\delta \mathbf{g}$ and $r=-q$, where

$$
\delta=\frac{1}{\alpha_{2}^{2}}\left(1+\frac{1}{\alpha_{1} \beta_{0}}\right)^{-1},
$$

then it follows that

$$
\begin{aligned}
& \mathscr{B}_{h}((\mathbf{v}, q) ;(\mathbf{w}, r)) \\
&=(\nabla \mathbf{v}, \nabla \mathbf{v})-\delta(\nabla \mathbf{v}, \nabla \mathbf{g})+\delta(q, \operatorname{div} \mathbf{g})+\beta \mathscr{C}_{h}(q, q) \\
& \geq|\mathbf{v}|_{1}^{2}-\delta|\mathbf{v}|_{1}|\mathbf{g}|_{1}+\delta\left(\Pi_{h} q, \operatorname{div} \mathbf{g}\right) \\
&-\delta\left(\left(\Pi_{h}-I\right) q, \operatorname{div} \mathbf{g}\right)+\alpha_{1} \beta\left\|\left(I-\Pi_{h}\right) q\right\|_{0}^{2} \\
& \geq|\mathbf{v}|_{1}^{2}-\delta \alpha_{2}|\mathbf{v}|_{1}\left\|\Pi_{h} q\right\|_{0}+\delta\left\|\Pi_{h} q\right\|_{0}^{2} \\
&-\delta \alpha_{2}\left\|\left(I-\Pi_{h}\right) q\right\|_{0}\left\|\Pi_{h} q\right\|_{0}+\alpha_{1} \beta_{0}\left\|\left(I-\Pi_{h}\right) q\right\|_{0}^{2} \\
& \geq \frac{1}{2}|\mathbf{v}|_{1}^{2}-\frac{\delta^{2} \alpha_{2}^{2}}{2}\left\|\Pi_{h} q\right\|_{0}^{2}+\delta\left\|\Pi_{h} q\right\|_{0}^{2} \\
&-\frac{\delta^{2} \alpha_{2}^{2}}{2 \alpha_{1} \beta_{0}}\left\|\Pi_{h} q\right\|_{0}^{2}+\frac{\alpha_{1} \beta_{0}}{2}\left\|\left(I-\Pi_{h}\right) q\right\|_{0}^{2},
\end{aligned}
$$


that is,

$$
\mathscr{B}_{h}((\mathbf{v}, q) ;(\mathbf{w}, r)) \geq \frac{1}{2}|\mathbf{v}|_{1}^{2}+\frac{\delta}{2}\left\|\Pi_{h} q\right\|_{0}^{2}+\frac{\alpha_{1} \beta_{0}}{2}\left\|\left(I-\Pi_{h}\right) q\right\|_{0}^{2},
$$

i.e., there is a positive constant $\kappa_{1}$, independent of $\beta$, such that

$$
\mathscr{B}_{h}((\mathbf{v}, q) ;(\mathbf{w}, r)) \geq \kappa_{1}\left(\|\mathbf{v}\|_{1}+\|q\|_{0}\right)^{2} \text {. }
$$

On the other hand,

$$
\|\mathbf{w}\|_{1}+\|r\|_{0} \leq \kappa_{2}\left(\|\mathbf{v}\|_{1}+\|q\|_{0}\right)
$$

for some positive constant $\kappa_{2}$.

Finally, combining (3.13) and (3.14) establishes inequality (3.10) with $\alpha=$ $\kappa_{1} / \kappa_{2}$, independent of $\beta$.

Below, $C_{1}, C_{2}, C_{3}, \ldots$ will be used to denote constants which are independent of $h$ and $\beta$. In the first instance, we let $\tilde{\mathbf{u}}$ denote the interpolant of $\mathbf{u}$ in $\mathbf{V}_{h}$, and $\tilde{p}$ the interpolant of $p$ in $P_{h}$. Then, for all $(\mathbf{v}, q) \in \mathbf{V}_{h} \times P_{h}$,

$$
\begin{aligned}
\mathscr{B}_{h} & \left(\left(\mathbf{u}_{h}-\tilde{\mathbf{u}}, p_{h}-\tilde{p}\right) ;(\mathbf{v}, q)\right) \\
\quad & =\mathscr{B}_{h}\left(\left(\mathbf{u}_{h}, p_{h}\right) ;(\mathbf{v}, q)\right)-\mathscr{B}((\tilde{\mathbf{u}}, \tilde{p}) ;(\mathbf{v}, q))+\beta \mathscr{C}_{h}(\tilde{p}, q) \\
& =\mathscr{B}((\mathbf{u}, p) ;(\mathbf{v}, q))-\mathscr{B}((\tilde{\mathbf{u}}, \tilde{p}) ;(\mathbf{v}, q))+\beta \mathscr{C}_{h}(\tilde{p}, q)
\end{aligned}
$$

$$
=\mathscr{B}((\mathbf{u}-\tilde{\mathbf{u}}, p-\tilde{p}) ;(\mathbf{v}, q))+\beta \mathscr{C}_{h}(\tilde{p}, q) .
$$

Using the stability inequality (3.10) in the usual way gives

$$
\begin{aligned}
\left\|\mathbf{u}_{h}-\tilde{\mathbf{u}}\right\|_{1}+\left\|p_{h}-\tilde{p}\right\|_{0} \\
\leq C_{1}\left(\|\mathbf{u}-\tilde{\mathbf{u}}\|_{1}+\|p-\tilde{p}\|_{0}+\beta \sup _{\substack{q \in P_{h} \\
\|q\|_{0}=1}} \mathscr{C}_{h}(\tilde{p}, q)\right) .
\end{aligned}
$$

In order to estimate the jump term in (3.16), note that, since we assume that $p \in H^{1}(\Omega)$, we have $\mathscr{C}_{h}(p, q)=0 \quad \forall q \in P_{h}$. Using the triangle and Schwarz inequalities (and making use of the regularity conditions (2.5) and (2.6)) then gives

$$
\begin{aligned}
\mathscr{C}_{h}(\tilde{p}, q) & =\mathscr{C}_{h}(\tilde{p}-p, q) \\
& \leq C_{2}\left(\sum_{K \in \tau_{h}} h_{K} \int_{\partial K}|p-\tilde{p}|^{2} d s\right)^{1 / 2}\left(\sum_{K \in \tau_{h}} h_{K} \int_{\partial K}|q|^{2} d s\right)^{1 / 2},
\end{aligned}
$$

where $\partial K$ refers to an element edge, and by a scaling argument (cf. [2, Lemma 1])

$$
\left(\sum_{K \in \tau_{h}} h_{K} \int_{\partial K}|q|^{2} d s\right)^{1 / 2} \leq C_{3}\|q\|_{0}
$$

implying that

$$
\sup _{\substack{q \in P_{h} \\\|q\|_{0}=1}} \mathscr{C}_{h}(\tilde{p}, q) \leq C_{2} C_{3}\left(\sum_{K \in \tau_{h}} h_{K} \int_{\partial K}|p-\tilde{p}|^{2} d s\right)^{1 / 2}
$$


Substituting into (3.16), and applying the triangle inequality in the usual way, gives

$$
\begin{aligned}
\| \mathbf{u} & -\mathbf{u}_{h}\left\|_{1}+\right\| p-p_{h} \|_{0} \\
& \leq C_{4}\left(\|\mathbf{u}-\tilde{\mathbf{u}}\|_{1}+\|p-\tilde{p}\|_{0}+\beta C_{5}\left(\sum_{K \in \tau_{h}} h_{K} \int_{\partial K}|p-\tilde{p}|^{2} d s\right)^{1 / 2}\right)
\end{aligned}
$$

with constants $C_{4}=1+C_{1}$ and $C_{5}=C_{1} C_{2} C_{3} /\left(1+C_{1}\right)$.

Using a further scaling argument (cf. [2, Lemma 3]) then gives the estimate

$$
\left(\sum_{K \in \tau_{h}} h_{K} \int_{\partial K}|p-\tilde{p}|^{2} d s\right)^{1 / 2} \leq C_{6} h|p|_{1},
$$

implying an optimal error estimate (3.9) except that the constant depends on $\beta$ (which implies that the approximation becomes increasingly inaccurate as $\beta$ is increased).

To see that the method does not actually behave in this way, consider replacing the interpolant $\tilde{p}$ in $(3.15)$ by $\tilde{\tilde{p}}$, the $L^{2}$ projection of $p$ into $Q_{h}$, giving

$$
\left\|\mathbf{u}_{h}-\tilde{\mathbf{u}}\right\|_{1}+\left\|p_{h}-\tilde{\tilde{p}}\right\|_{0} \leq C_{1}\left(\|\mathbf{u}-\tilde{\mathbf{u}}\|_{1}+\|p-\tilde{\tilde{p}}\|_{0}+\beta \sup _{\substack{q \in P_{h} \\\|q\|_{0}=1}} \mathscr{C}_{h}(\tilde{\tilde{p}}, q)\right)
$$

In this case, we have $\mathscr{C}_{h}(\tilde{\tilde{p}}, q)=0 \quad \forall q \in P_{h}$, implying the error estimate

$$
\left\|\mathbf{u}-\mathbf{u}_{h}\right\|_{1}+\left\|p-p_{h}\right\|_{0} \leq C_{4}\left(\|\mathbf{u}-\tilde{\mathbf{u}}\|_{1}+\|p-\tilde{\tilde{p}}\|_{0}\right) \text {. }
$$

Combining (3.17) with (3.19) gives the "optimal error estimate"

$$
\begin{array}{r}
\left\|\mathbf{u}-\mathbf{u}_{h}\right\|_{1}+\left\|p-p_{h}\right\|_{0} \\
\leq C_{4}\left(\|\mathbf{u}-\tilde{\mathbf{u}}\|_{1}+\min \left\{\|p-\tilde{p}\|_{0}+\beta C_{5}\left(\sum_{K \in \tau_{h}} h_{K} \int_{\partial K}|p-\tilde{p}|^{2} d s\right)^{1 / 2},\right.\right. \\
\left.\left.\|p-\tilde{\tilde{p}}\|_{0}\right\}\right),
\end{array}
$$

proving that (3.9) is meaningful even in the limit of arbitrarily large $\beta$.

A feature of the proof of the theorem is the fact that it establishes the validity of the local stabilization approach with an arbitrarily large stabilization parameter. The method behaves like a constant pressure approximation over the macroelement in this case. It is easily seen that global stabilization methods will not be as robust, i.e., the constant in the error estimate in [9] tends to infinity as the "global" stabilization parameter is increased. In our other publications $[11,14]$, the numerical performance of the locally stabilized methods above is compared with that of the analogous globally stabilized methods of [9], and the inferior robustness of the globally stabilized methods is discussed in 
detail. The crucial point is made that when using a locally stabilized method, the magnitude of $\beta$ can be tuned to improve the rate of convergence of the iterative solver, without adversely affecting accuracy.

Corollary 3.1. Constructing a grid of $Q_{1}-P_{0}$ quadrilaterals such that the reference macroelement $\widehat{M}$ and an arbitrary $M \in \mathscr{E}_{\widehat{M}}$ are as illustrated in Figure 1 , leads to a stable mixed approximation satisfying the optimal error estimate

$$
\left\|\mathbf{u}-\mathbf{u}_{h}\right\|_{1}+\left\|p-p_{h}\right\|_{0} \leq O(h) .
$$

We note that the reference $2 \times 2$ macroelement is the obvious choice, since it is the smallest patch of elements which satisfies the macroelement connectivity condition (3.8).

Remark 3.1. The corresponding three-dimensional element is the $Q_{1}-P_{0}$ (trilinear velocity, constant pressure) hexahedron, which can be stabilized locally over a $2 \times 2 \times 2$ macroelement. Further details can be found in [10, Chapter 3].

Remark 3.2. The stability of the $2 \times 2$ macroelement above is not surprising, since it is well known that the standard $Q_{1}-P_{0}$ method can be rendered stable over a grid of $2 \times 2$ rectangular macroelements by excluding the local "checkerboard" pressure component from the pressure space (see for example, Gunzburger [8, p. 30]).

A similar stabilization of the $Q_{1}-P_{0}$ method above (also based on a $2 \times$ 2 macroelement construction), has earlier been proposed by Pitkäranta and Saarinen in [12]. Their method corresponds to (3.6) with a $\mathscr{C}_{h}$ defined by

$$
\mathscr{C}_{h}(r, q)=\left(\Pi_{h}^{c} r, \Pi_{h}^{c} q\right),
$$

where $\Pi_{h}^{c}$ denotes the orthogonal projection onto the space spanned by the checkerboard functions. For details see [12]. We note that the error estimate for the Pitkäranta and Saarinen method is identical to that above. The main advantage of our method is that it seems to be more straightforward to implement.

Corollary 3.2. Constructing a grid of $P_{1}-P_{0}$ triangles such that all the macroelements are equivalent to the reference macroelement $\widehat{M}$ illustrated in Figure 2 , also leads to a stable mixed approximation satisfying the optimal error estimate (3.20).

\section{BIBLIOGRAPHY}

1. I. Babuška, Error bounds for finite element method, Numer. Math. 16 (1971), 322-333.

2. I. Babuška, J. Osborn, and J. Pitkäranta, Analysis of mixed methods using mesh dependent norms, Math. Comp. 35 (1980), 1039-1062.

3. J. Boland and R. A. Nicolaides, Stability of finite elements under divergence constraints, SIAM J. Numer. Anal. 20 (1983), 722-731.

4. J. H. Bramble and J. E. Pasciak, A preconditioning technique for indefinite systems resulting from mixed approximations of elliptic problems, Math. Comp. 50 (1988), 1-17.

5. F. Brezzi and J. Pitkäranta, On the stabilisation of finite element approximations of the Stokes problem, Efficient Solutions of Elliptic Systems (W. Hackbusch, ed.), Notes on Numerical Fluid Mechanics, vol. 10, Vieweg, Braunschweig, 1984, pp. 11-19. 
6. J. Douglas, Jr. and J. Wang, An absolutely stabilized finite element method for the Stokes problem, Math. Comp. 52 (1989), 495-508.

7. V. Girault and P. A. Raviart, Finite element methods for Navier-Stokes equations: theory and algorithms, Springer-Verlag, Berlin, Heidelberg, 1986.

8. M. D. Gunzburger, Finite element methods for viscous incompressible flows, Academic Press, London, 1989.

9. T. J. R. Hughes and L. P. Franca, A new finite element formulation for CFD: VII. The Stokes problem with various well-posed boundary conditions: Symmetric formulations that converge for all velocity/pressure spaces, Comput. Methods Appl. Mech. Engrg. 65 (1987), 85-96.

10. N. Kechkar, Analysis and application of locally stabilised mixed finite element methods, Ph.D. Thesis, University of Manchester Institute of Science and Technology, 1989.

11. N. Kechkar and D. J. Silvester, The stabilisation of low order mixed finite elements for incompressible flow, Proc. 5th Internat. Sympos. on Numerical Methods in Engineering (R. Gruber et al., eds.), vol. 2, Computational Mechanics Publications, Southampton, 1989, pp. 111-116.

12. J. Pitkäranta and T. Saarinen, A multigrid version of a simple finite element method for the Stokes problem, Math. Comp. 45 (1985), 1-14.

13. R. L. Sani, P. M. Gresho, R. L. Lee, and D. F. Griffiths, The cause and cure (?) of the spurious pressures generated by certain finite element method solutions of the incompressible Navier-Stokes equations, Parts 1 and 2, Internat. J. Numer. Methods Fluids 1 (1981), 17-43; 171-204.

14. D. J. Silvester and N. Kechkar, Stabilised bilinear-constant velocity-pressure finite elements for the conjugate gradient solution of the Stokes problem, Comput. Methods Appl. Mech. Engrg. 79 (1990), 71-86.

15. R. Stenberg, Analysis of mixed finite elements for the Stokes problem: A unified approach, Math. Comp. 42 (1984), 9-23.

16. R. Verfürth, A multilevel algorithm for mixed problems, SIAM J. Numer. Anal. 21 (1984), 264-271.

Department of Mathematics, University of Manchester Institute of Science and TeChNology, P. O. BoX 88, MANChester M60 1QD, UNited Kingdom

E-mail address, D. Silvester: na.silvester@na-net.ornl.gov 